\title{
Three-Dimensional Imaging in Polymer Science: Its Application to Block Copolymer Morphologies and Rubber Composites
}

\author{
Hidehiko DohI, ${ }^{1}$ Hideaki Kimura, ${ }^{1}$ Marina Kotani, ${ }^{1}$ Takeshi KaneKo, ${ }^{2}$ \\ Tatsuro KITAOKA, ${ }^{2}$ Toshio NiSHI, ${ }^{3}$ and Hiroshi JINNAI ${ }^{2, \dagger}$ \\ ${ }^{1}$ SRI Research \& Development Ltd., Chuo-ku, Kobe 651-0071, Japan \\ ${ }^{2}$ Department of Macromolecular Science and Engineering, Graduate School of Science and Technology, \\ Kyoto Institute of Technology, Matsugasaki, Kyoto 606-8585, Japan \\ ${ }^{3}$ Department of Organic and Polymeric Materials, School of Science and Engineering, \\ Tokyo Institute of Technology, Meguro-ku, Tokyo 152-8552, Japan
}

(Received March 9, 2007; Accepted April 17, 2007; Published June 12, 2007)

\begin{abstract}
New methods to visualize polymer morphologies in three-dimension (3D) in polymer science are reviewed. Here we concentrate on one of such 3D imaging technique, transmission electron microtomography (TEMT), and introduce some experimental studies using this novel technique. They are block copolymer morphologies during order-order transition between the two different morphologies and block copolymer thin film morphology also during morphological change due to confinement. Direct visualization of 3D structure of silica particle/rubber composite and related morphological analyses are shown. Subsequently, as a very hot topic of the 3D imaging, we show for the first time to characterize the morphological change in a silica particle/rubber composite upon stretching. It was found that the aggregates of silica particles were broken down upon stretching and many voids were generated near and between the silica particles. Local stress upon stretching inside the composite was inferred from the image intensity of the 3D reconstructed image. The local stress was found not only near the silica particles but also near the top of the voids. The observations indicated that the local stress increases the modulus, causing voids to form along the stretching direction. The thickness of the specimen after the stretching was also estimated from the 3D volume data, which turned out to be non-uniform and thinner than what is expected from the affine deformation. These experimental findings indicate that the rubber composite does not obey the assumption of the affine deformation at the nano-scale.

[doi:10.1295/polymj.PJ2006259]

KEY WORDS Transmission Electron Microtomography / Three-dimensional Imaging / Orderorder Transition / Stretching / Silica / Rubber Composite / Local Strain in Rubber Matrix /
\end{abstract}

Polymer materials are ubiquitous in our daily life. Such materials often consist of more than one species of polymers and thus become multi-component systems, such as in polymer blends, ${ }^{1,2}$ block copolymers, ${ }^{3}$ and fillers/polymer composites. ${ }^{4}$ The multi-component systems often show phase-separated structures due to immiscibility of constituents. Studies to characterize such morphologies inside the materials have been growing intensively over the past few decades. Academic interest in complex fluids (to which polymeric systems belong) as well as the ceaseless industrial need for developing new materials motivates such studies.

In academia, pattern formation and self-assembling processes of multi-component polymer systems are one of the most fascinating research themes in nonlinear, non-equilibrium phenomena. ${ }^{1}$ Likewise, nanometer-scale periodic structures formed in block copolymers in their equilibrium state are interesting because their self-assembly is driven by the subtle balance between the entropic block chain conformation and enthalpic interaction between the constituents. ${ }^{3}$ In industry, phase-separated polymer systems provide an important route to achieve superior physical properties. Hence, the structure-property relationship in multi-component polymeric materials is of significant importance, basic studies on which eventually render new designs of polymer materials satisfying the diverse requirements of industry.

Filler/polymer composites are another key class of polymer materials. Incorporation of a few percent of clay minerals into a polymer matrix in many cases synergistically increases the modulus, gas barrier properties, heat distortion temperature, etc. It is also well known that the improvement of such properties strongly depends on structures of fillers inside the polymer matrix. ${ }^{5}$ As typical fillers, carbon black (CB), silica particles, clay mineral are often used. As

${ }^{\dagger}$ To whom correspondence should be addressed (Tel: +81-75-724-7846, Fax: +81-75-724-7770, E-mail: hjinnai@kit.ac.jp). 
many different types of polymers can be used as the matrix, rubber is one of the most common polymers in industrial use. For example, the $\mathrm{CB} /$ rubber composites are often used as composite-type materials that are made of polymers, fillers, cross-linking agents, and other additives. While filler particles are known to alter the mechanical properties of rubber materials, the mechanism by which the alterations occur is still a subject of debate. ${ }^{6}$ Today, extensive efforts are being made to unravel the mechanism of rubber reinforcement by fillers and the fracture process of rubber vulcanizates. A complete fundamental understanding of this complex mechanism remains a significant scientific challenge. ${ }^{7-14}$

\section{THREE-DIMENSIONAL (3D) IMAGING}

In order to establish the structure-mechanical properties relationship, one needs proper methods to characterize the morphologies. Up to now, morphological studies of the polymeric materials have been carried out by various microscopic and scattering methods. Optical microscopes, transmission electron microscopes (TEM), scanning electron microscopes (SEM) and atomic force microscopes (AFM) are commercially available and widely used. The biggest advantage to use such microscopes is that they offer intuitive real-space representation of the morphologies. However, when it comes to "measurements", especially in a quantitative way, the microscopes may give only limited pieces of structural information with inferior statistical accuracy to scattering methods. Scattering techniques give statistically-averaged structural information over the scattering volume which can be several orders of magnitude larger than the volume probed by the microscopes. Moreover, the scattering methods do not directly provide intuitive insight into the morphologies. The scattering methods often require knowledge of the morphologies beforehand to obtain the structural parameters: The two methods are complementary.

In some cases, however, identification of complex structures by the above microscopes is inconclusive and therofore the interpretation of scattering data becomes quite difficult. One of the reasons is that the microscopes used so far take two-dimensional (2D) (transmitted or surface) images of three-dimensional (3D) objects. The more complicated the morphologies are, the less convincing the 2D images usually become. It is thus requisite to develop microscopes that are capable of visualizing 3D morphologies in 3D. A couple of different types of 3D microscopes have been developed. ${ }^{15}$ They are laser scanning confocal microscopy (LSCM), ${ }^{16-27} \mathrm{X}$-ray tomography, ${ }^{28-30}$ and transmission electron microtomography (TEMT). ${ }^{15,31-54}$
The spatial resolution of LSCM and X-ray tomography is on the order of a micrometer, while that of TEMT approaches $1 \mathrm{~nm}$ or less. ${ }^{44,52}$ We also emphasize that 3D microscopy should be accompanied by digital image analyses that extract useful and new structural information from the $3 \mathrm{D}$ volume data. ${ }^{55-58}$

Among those 3D imaging techniques, with rapid advances in "nanotechnology", there is a growing need for quantitative structural analysis at the nanometer scale. In TEMT, a series of projections at different tilt angles are obtained, which are subsequently used to calculate tomograms on the basis of computerized tomography (CT). In the coarse of the "reconstruction", precise alignment of projections to a tilt axis is required in order to obtain high-resolution 3D data. This alignment should be the foundation for the subsequent data analysis to extract various kinds of structural information. Another critical issue associated with TEMT is what is called the "missing wedge" problem: Image quality of tomograms is considerably deteriorated due to the limitation of the tilting angle up to $\pm 60 \sim 70^{\circ}$ in the transmission electron microscope. Kawase et al. recently demonstrated a protocol to perform complete rotation, i.e., $\pm 90^{\circ}$, of their $\mathrm{ZrO}_{2} /$ polymer nanocomposite, by which they achieved truly quantitative TEMT for the first time. ${ }^{52,59}$ The detailed theoretical backgrounds as well as experimental results are described in a separate review article. ${ }^{15}$

\section{SOME APPLICATIONS USING TEMT}

\section{D Morphological Studies of Block Copolymers}

TEMT has been used to characterize 3D structures in a wide range of materials. ${ }^{15,39}$ As described above, block copolymers self-assemble into various periodic nano-scale structures ("microphase-separated structures") and hence their 3D morphologies are subjects for many scientific studies. In 1988, Spontak and coworkers $^{32}$ used TEMT to observe cylindrical morphologies of block copolymer for the first time. Later, a complex morphology, Gyroid, has been extensively studied using TEMT. ${ }^{35,37}$ Complicated 3D "maze" was beautifully imaged (see, for example, Figure 1 in ref 37), from which some structural features, e.g. volumetric fraction of one of the constituents, interfacial curvatures,${ }^{55,56}$ topology, ${ }^{60}$ connectivity of domains ${ }^{57,58}$ etc. were measured.

The grain boundary of microphase-separated structure is another important aspects of block copolymer morphologies. The grain boundary morphology of lamellar structure where two lamellae intersect orthogonally was examined by TEMT (see Figure 5 in reference 44). It was found that the lamellae microdomains were continuous through the boundary region and the 
boundary morphology had characteristics of the saddle-like hyperbolic surface, called the Scherk's first surface. This experimental result demonstrates that the minimization of the surface area, i.e., the minimization of the surface energy, is the dominant cause of the structural formation. ${ }^{44}$ Another intriguing boundary structure was found between the Gyroid and hexagonally-perforated lamellar (HPL) morphologies during the order-order transition. ${ }^{61}$ Figure 1(a) shows a 3D image of the boundary between these two morphologies where every other HPL layers were beautifully connect to one of the two non-intersecting Gyroid networks. Moreover, as clearly shown in Figure 1(b), nature of epitaxy can be found: $\{1 \overline{1} 1\}$, $\{20 \overline{2}\}$ and $\{121\}$ planes were at the boundary with the $\{121\}$ plane being parallel to the HPL layers.

TEMT is also useful to investigate microphase-separated structures of block copolymer thin films. Figure 2 demonstrates order-order transition induced by the confinement (finite thickness of the film) where the cylindrical morphology transformed into spherical morphology upon annealing. ${ }^{62}$ Note that the equilibrium structure of this block copolymer in the bulk state is cylindrical morphology. The stability of either cylinders or spheres in the block copolymer thin film was simulated on the basis of the Self Consistent Field Theory, which is in excellent agreement with the experimental results. ${ }^{62}$

\section{D Morphological Studies of Composite Materials}

Figure 3 shows a 3D structure of a rubber composite consisting of silica particles in rubbery matrix. Figure 3(a) and 3(b) display a TEM micrograph and corresponding TEMT image, respectively. The TEMT image contains richer structural information than the 2D TEM image. Using a Monte-Carlo simulation, spatial position of each silica particle was resolved ${ }^{63,64}$ and displayed in Figure 3(c). Note that this protocol is necessary to evaluate 3D spatial distribution, e.g., the spatial correlation function of fillers, especially when individual fillers are too small to be clearly visualized but only regions of the fillers are obtained. The simulated silica particles can be further used to measure the persistent length of fillers as demonstrated in Figure 3(d). ${ }^{65}$ Needless to say, the persistent length of fillers should be an important measure to understand electrical property of semi-conductive materials, e.g., $\mathrm{CB} /$ rubber composites. Besides these structural parameters, distribution and orientation of filler particles $^{45}$ have been discussed. We note here that most of this structural information can never be obtained from other existing experimental techniques. ${ }^{15}$ Although TEMT is receiving significant attention in polymer science, the 3D imaging of strain under an external field, e.g., strain, has never been carried out.
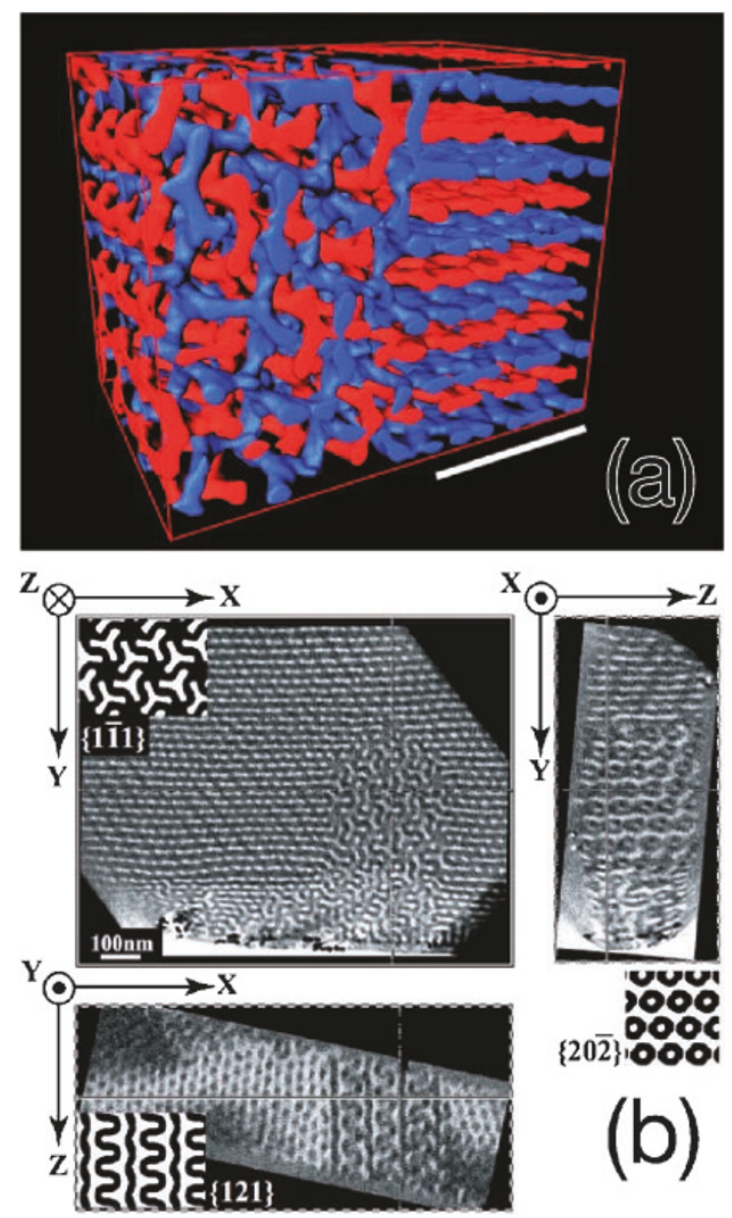

Figure 1. (a) 3D morphology of Poly(styrene-block-isoprene) (SI) block copolymer during order-order transition (OOT) from hexagonally-perforated lamellar (HPL) structure (right) to Gyroid structure (left). ${ }^{62}$ The Gyroid structure has Ia $\overline{3} \mathrm{~d}$ symmetry. The number-averaged molecular weight and polydispersity index are $M_{\mathrm{n}}=37,000$ and $M_{\mathrm{w}} / M_{\mathrm{n}}=1.01$. The volume fraction of polyisoprene is 0.62 . Blue and red microdomains consist of polystyrene and continuous through the boundary. Non-intersecting PS microdomains independently and periodically connect to the HPL layers. Scale bar is $100 \mathrm{~nm}$. (b) Orthogonal views of the $3 \mathrm{D}$ reconstruction of SI block copolymer during OOT. Z-axis is parallel to the optical axis of the microscope and also the depth direction of the sample. As shown in each cross-section, the PS microdomain (white domain) of the Gyroid exhibits characteristic crystallographic planes, demonstrating that the OOT is epitaxial. The insets in the cross-sections show characteristic patterns of Gyroid viewed from certain crystallographic planes indicated by the miller numbers.

\section{D Morphologies of Filler/Rubber Composite Upon Stretching}

In order to establish the structure-property (mechanical property) relationship, complete understanding of the phenomena occurring under strain ${ }^{66}$ is crucial. Such studies will provide new insight on the nano-scale structures in rubber that cannot be obtained by observing rubber in the static condition. We report here, as a first attempt, the analytical results of the 3D 

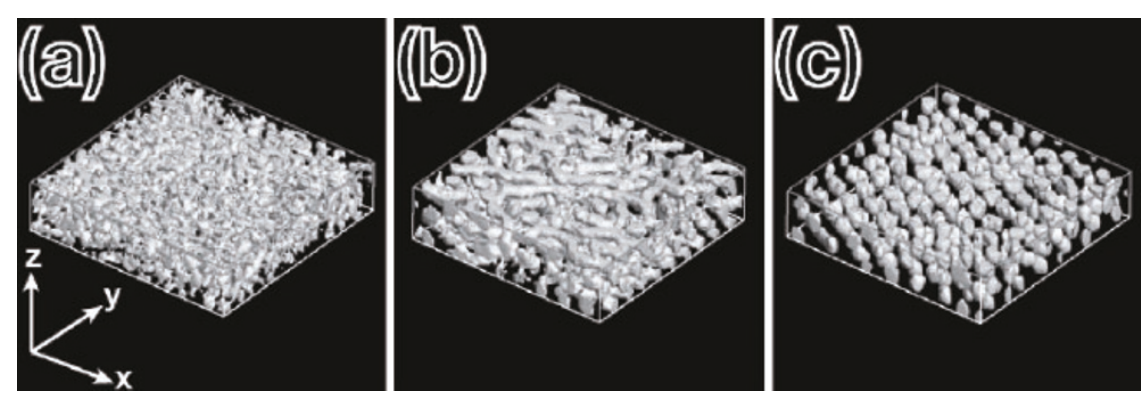

Figure 2. 3D morphologies of Poly(styrene-block-isoprene) (SI) block copolymer thin film. ${ }^{62}$ The number-averaged molecular weight and polydispersity index are $M_{\mathrm{n}}=51,000$ and $M_{\mathrm{w}} / M_{\mathrm{n}}=1.06$, respectively. The volume fraction of polyisoprene is 0.22 . A specimen film was prepared by spin-coating from toluene solutions with $2.6 \mathrm{wt} \%$ onto the mica substrate. Microphase-separated structures of (a) as-spun film and annealed at $120^{\circ} \mathrm{C}$ for (b) 2880 minutes ( $48 \mathrm{~h}$ ), (c) 9000 minutes $(150 \mathrm{~h})$ under vacuum. Polyisoprene phase is shown Box size: $300 \times 300 \times 70 \mathrm{~nm}$.
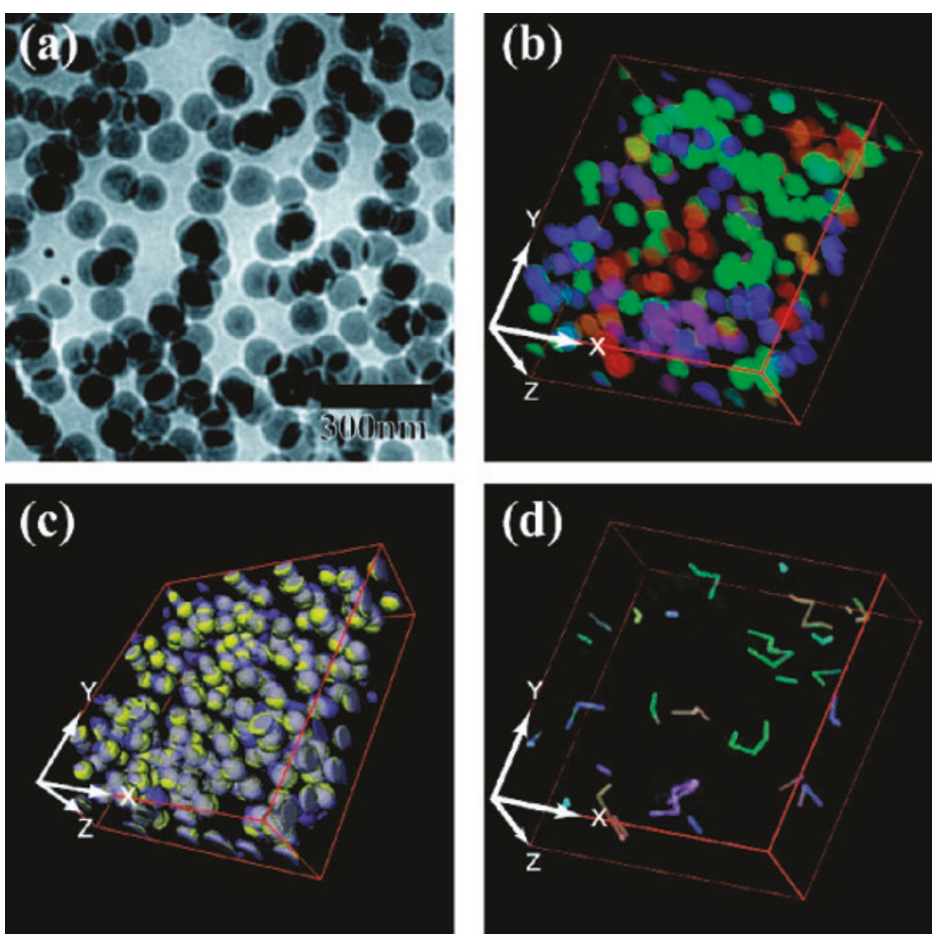

Figure 3. (a) TEM micrograph of silica particle/rubber composite. ${ }^{64,65}$ An emulsion-type styrene-butadiene rubber (SBR1502, Zeon Corp. (Japan)) was used as matrix rubber. Mixture of SBR and silica particles with small amount of additives, i.e., sulfer, N-(benzo[d]thiazol-2-ylthio)-2-methylpropan-2-amine (TBBS), Si266 (Bis (triethoxysilylpropyl) disulfide) was compounded in an 8-inch open roll mixer. The mixing was continued for approximately $5 \mathrm{~min}$. The compounded mixtures were then thermally treated at $170{ }^{\circ} \mathrm{C}$ for $10 \mathrm{~min}$ in a hot press for vulcanization. The volume content of silica (Seahoster KE-P1, diameter $100 \mathrm{~nm}$ ) was $20 \%$. The 3D picture obtained from TEMT is shown in part (b) (box size: $1.32 \mu \mathrm{m} \times 1.32 \mu \mathrm{m} \times 411 \mathrm{~nm}$ ). Silica particles with same color belong to same aggregate. Spheres with diameter of $100 \mathrm{~nm}$ were embedded by a monte-carlo simulation as shown in part (c), in which transparent blue and solid yellow spheres correspond, respectively, to experimentally-visualized silica particles and simulated particles. Persistent length in each aggregate was estimated and is shown in part (d) by solid line. TEM and TEMT pictures were taken on a JEOL JEM-2200FS.

morphological change in stretched rubber. By the 3D observation of stretched rubber specifically the observation of the local stresses and voids inside the stretched rubber, we can determine these mechanisms in rubber composite.

Figure 4(a) shows the 3D image of silica particles in cured rubber before stretching. Some silica particles tend to form aggregates, however they do not aggre- gate to the extent observed in carbon black particles (e.g., N220). This is because the bonding force between the silica particles is not as large as that between carbon black particles. There are no appreciable voids or cracks in the specimen prior to stretching.

Figure 4(b) shows the 3D image of the silica particles in cured rubber after 100\% stretching (extension ratio $\alpha=2$ ). Note that the $3 \mathrm{D}$ images shown in 

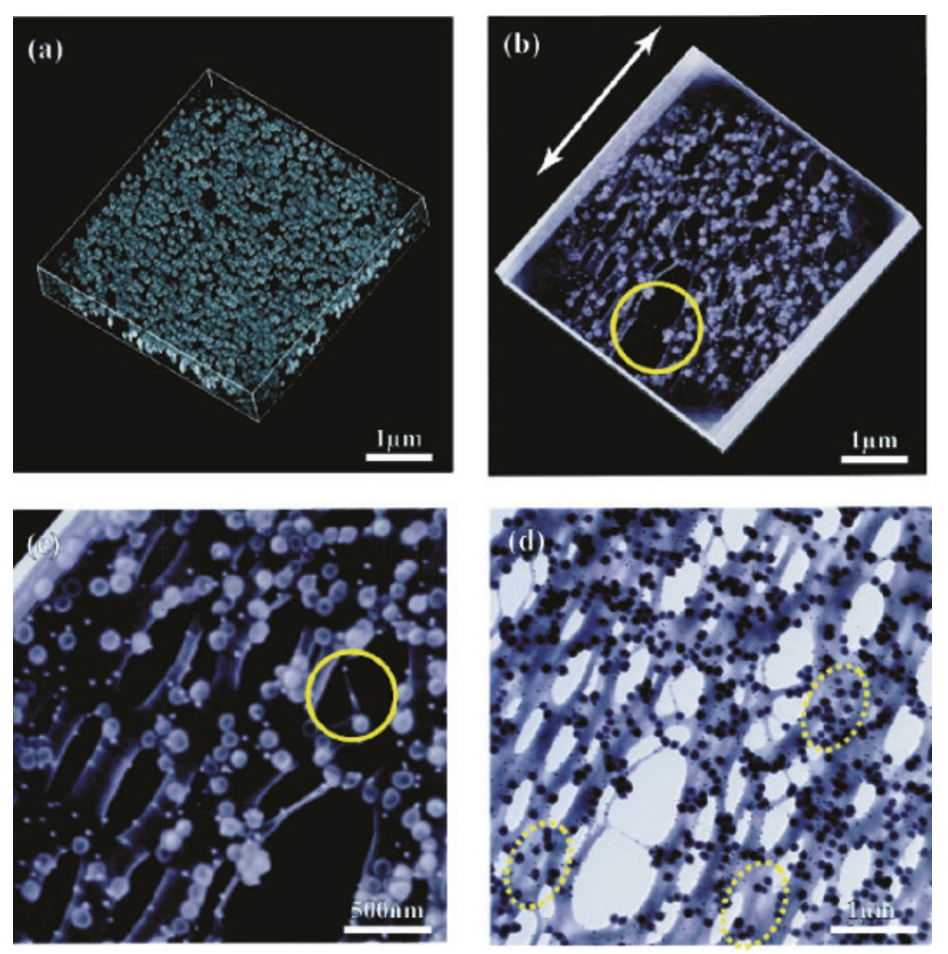

Figure 4. TEMT 3D reconstructed image of (a) unstretched and (b) stretched states of silica particle/rubber composite as used in Figure 3. White spheres in part (a) to (c) are silica particles. Extension ratio is 2. Stretched direction is shown by the white arrow in part (b). Magnified view of the 3D reconstruction is shown in part (c). After stretching, many voids are observable, in which "polymer bridges" are discernible (indicated by circles in part (b) and (c)). TEM micrograph corresponding to the volume shown in part (b) is shown in part (d). For observation under stretching, the specimen was set on the copper grid and stretched uniaxially. ${ }^{7,68-71}$

Figure 4(a) and (b) were taken using different cryomicrotomed sections and thus they do not represent the same field of view. The distance between the silica particles increased as the specimen was stretched. Most of the silica aggregates were broken down upon stretching, while some remained. This suggests that the bonding force between some silica particles was stronger than the stress induced by $100 \%$ elongation. Many voids were generated near and between the silica particles. The voids tended to be generated between two silica particles and connect them. They formed lines along the stretching direction; these voids are $90-2000 \mathrm{~nm}$ long and $40-800 \mathrm{~nm}$ wide. Moreover, we found that fine rubber polymer existed in some voids: these polymers are $70-440 \mathrm{~nm}$ long and 5-70 nm wide. Since these polymers strands appear to "bridge" the voids, we hereafter refer to them as "polymer bridges." These polymer bridges are similar to fibrils found in crazing. It is very likely that the polymer bridges were made up of stretched and assembled polymer chain, which implies that, although nearly all the polymer chains on both sides of the polymer bridge were broken down, these assembled polymer chains did not snap and remained in the void space. Figure 4(c) shows the magnified 3D images of the stretched rubber. The voids that connect the silica particles and the polymer bridges are clearly visible.
Figure 4(d) shows the 2D TEM image of the specimen. Here, we cannot distinguish between the silica particles on the rubber surface and those inside the rubber matrix. Although the $2 \mathrm{D}$ projection does not provide any information along the depth of the section, we did find areas that appeared to be a little pale, especially near the silica particles, in the rubber matrix. We hereafter call these areas "half-bright zones". The half-bright zones appeared to be located along the stretching direction, some of which are indicated by dashed circles in Figure 4(d). The brightness of the TEM image roughly corresponds to the density of the specimen. Therefore, the thickness of a half-bright zone may be thinner than that of the gray zone. This observation indicates that the rubber elongation in the half-bright zone is larger than that of the gray zone and that local stress occurs at the half-bright zone upon stretching.

Figure 5(a) and (b) show the horizontal cross-sectional images (XY plane) before and after stretching, respectively. Note that the electron beam was irradiated along the Z-axis, the axis along the thickness of the specimen. These cross-sectional images can be acquired by slicing an arbitrary plane of the 3D image. There exist many voids, polymer bridges, and halfbright zones showing the local stress upon stretching (Figure 5(b)). 

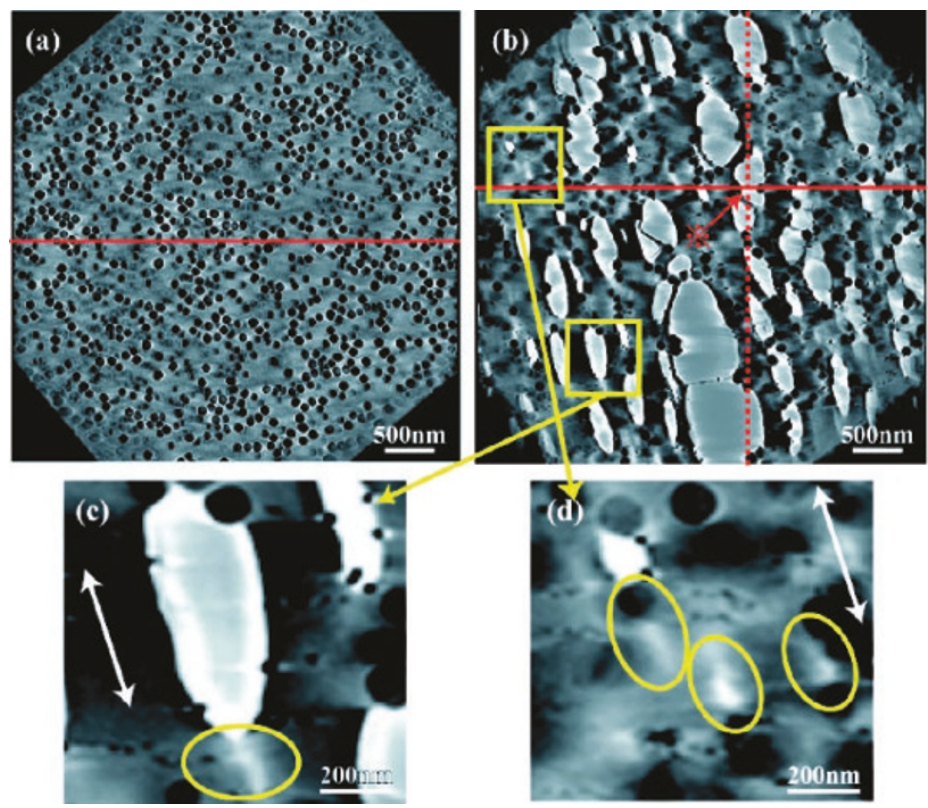

Figure 5. Digitally-sliced cross-sectional image of TEMT 3D reconstructed images of silica/rubber composite. (a) unstretched and (b) stretched states. Thickness of the digitally-sliced cross-section is $5.2 \mathrm{~nm}$. Black spheres in the cross sections represent silica particles. In part (b), white arrow is a stretching direction. Many voids are observable. Part (c) and (d) show magnified view of rectangles in part (b). As indicated by circles, there are half-bright zones that may be characteristic to the local stress upon stretching. ${ }^{66}$

Comparing Figure 4(d) (2D image) and Figure 5(b) (3D XY plane), we can see that the contrast of the XY plane is greater than that of the $2 \mathrm{D}$ image. We find that there are more half-bright zones in the XY digital slice. They tend to align along the stretching direction, indicating that the local stress also occurs near the top of the voids. ${ }^{67}$ The local stress near the top of voids must be the point from which void propagation originates. Figure 5(c) and (d) show the magnified images of the local stress. TEM images include all the information along the depth showing average image brightness of the specimen. On the contrary, the TEMT digital slice can provide an arbitrary cross-sectional image that provides information regarding the limited thin section (in this study, the thickness is $5.2 \mathrm{~nm}$ ). Thus, we can acquire a high-contrast image using the cross-sectional TEMT image.

The thickness of each specimen was measured from the 3D reconstruction in the following way. We randomly selected 10 points in the XY plane. For each point, uppermost and bottommost edges of the specimen were found by scanning XY digital slices along the $\mathrm{Z}$ direction. The sample thickness was determined by averaging these 10 thicknesses. The thicknesses before and after stretching were approximately 400$460(424) \mathrm{nm}$ and 70-240 (182) nm, respectively (numbers in the parentheses are average values). It was found that the thickness of the specimen decreases and the variation of thickness increases upon stretching. If the specimen obeyed affine deformation, the thickness should be in the range from $400 \div \sqrt{2}=$
$280 \mathrm{~nm}$ to $460 \div \sqrt{2}=330 \mathrm{~nm}$. Thus, it is evident that the thickness deformation of rubber composite does not conform to affine deformation. This result might be caused by the local stress and void generation in the specimen.

Figure 6(a), (b), and (c) show the schematic representation of the vertical cross section before and after stretching. Location of these cross sections are indicated in Figure 5(a) and (b) by solid lines. These figures clearly demonstrated that the thickness of the specimen decreased with stretching, while the variation of thickness increased with stretching. The fine rubber polymer (polymer bridge) was also observed. The thickness of the polymer bridge was approximately $70 \mathrm{~nm}$, which is approximately one-sixth of the thickness before stretching. This result indicates that the polymer chains are highly strained at the polymer bridges.

Let us now consider the fracture process of the rubber materials containing inorganic particles upon stretching. The local stress, which increased modulus and hardness, first occurred at the interface of the particles and rubber polymer. Upon further stretching, the detachment between the particle surface and polymer took place. Nanovoids were generated at the interface between the particles and rubber. The local stress near the top of the voids caused the voids to grow. The voids grew and became larger with stretching, and finally connected between two particles. This is the fracture process of stretched rubber containing inorganic particles. The results obtained from this study 


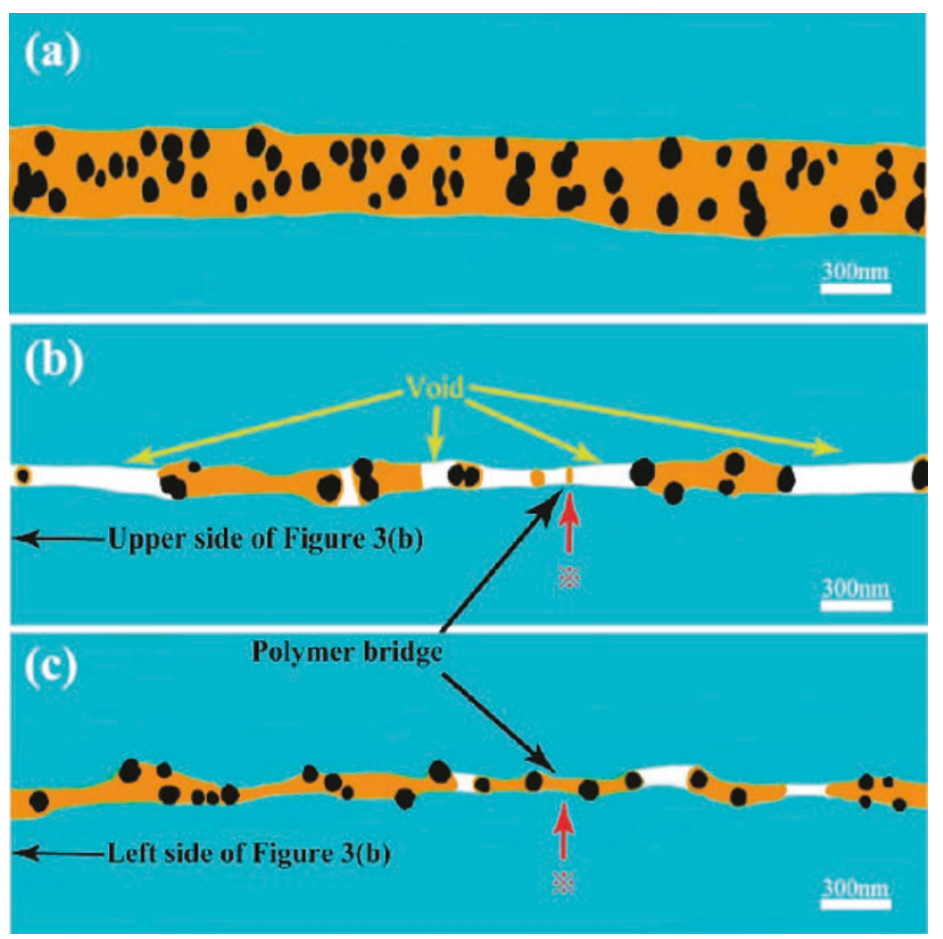

Figure 6. Schematic representations of cross-section of silica particle/rubber composite. Part (a) shows the cross-section at the position indicated by red line in Figure 5(a). Black spheres (silica particle) dispersed in ocher zone (rubber matrix). Part (b) and (c) correspond to cross-sections of stretched composite shown by dashed and solid lines in Figure 5(b), respectively. Voids viewed from side of the cryomicrotomed section are indicated by white areas. ※ represents the same position in Figure 5(b).

suggest the fundamental mechanism of reinforcement and fracture of the rubber composite.

Acknowledgment. HD is grateful to Prof. Tetsuo Asakura, Department of Biotechnology, Tokyo University of Agriculture and Technology, for useful discussions with regard to this study. The authors also wish to thank Dr. Eiko Nakazawa, Hitachi High-Technologies, for the preliminary test of stretched silica/ rubber samples and also acknowledge Mr. Hirofumi Nakamae, Mr. Naoki Tsukamori, Mr. Masaya Sakai and Mr. Hiroyuki Kishimoto, SRI R\&D Ltd., for their cooperation in this work. HJ gratefully acknowledge Prof. Taihyun Chang and Mr. Hae-Woong Park (Pohang University of Science and Technology), Prof. Masao Doi and Dr. Hiroshi Morita (The University of Tokyo) and Mr. Kohji Sawa (KIT) by their helpful discussions. HJ is also grateful to NEDO for support through the Japanese National Project "Nano-Structured Polymer Project" by the Ministry of Economy, Trade and Industry and for support from the Ministry of Education, Science, Sports and Culture through Grant-in-Aid No. 1855019 and No. 19031016.

\section{REFERENCES}

1. J. D. Gunton, M. S. Miguel, and P. S. Sahni, in "Phase transition and critical phenomena," C. Domb, and J. L.
Lebowitz, Ed., Academic, New York, 1983.

2. T. Hashimoto, in "Material science and technology," R. W. Cahn, P, Haasen, and E. J. Kramer, Ed., VCH, Weinheim, 1993, vol. 12, p 251.

3. V. Abetz and P. F. W. Simon, in "Block Copolymers I," V. Abetz, Ed., Springer, Heidelberg, 2005.

4. "Polymer-Clay Nanocomposites," T. I. Pinnavaia and G. W. Beale, Ed., John Wiley, New York, 2000.

5. S. S. Ray, K. Okamoto, and M. Okamoto, Macromolecules, 36, 2355 (2003).

6. J. S. Bergstöm and M. C. Boyce, Rubber Chem. Technol., 72, 633 (1999).

7. W. M. Hess, F. Lyon, and K. A. Burgess, Kautsch. Gummi Kunstst., 20, 135 (1967).

8. A. R. Payne and R. E. Whittaker, Rubber Chem. Technol., 44, 440 (1971).

9. T. Nishi, J. Polym. Sci., Polym. Phys. Ed., 12, 685 (1974).

10. A. N. Gent and B. Park, J. Mater. Sci., 19, 1947 (1984).

11. Y. Fukahori and W. Seki, Polymer, 33, 502 (1992).

12. Y. Fukahori and W. Seki, J. Materi. Sci., 28, 4143 (1993).

13. N. Suzuki and M. Ito, e-J. Soft Mater., 1, 1 (2005). (URL: http://www.jstage.jst.go.jp/article/ejsm/1/0/1_1/_article/-char/ $\mathrm{ja} /)$.

14. G. Heideman, R. N. Datta, and J. W. M. Nordermeer, Rubber Chem. Technol., 77, 512 (2004).

15. H. Jinnai, Y. Nishikawa, T. Ikehara, and T. Nishi, $A d v$. Polym. Sci., 170, 115 (2004).

16. T. Wilson, in "Confocal microscopy," T. Wilson, Ed., Academic, London, 1990.

17. H. Verhoogt, J. van Dam, A. Posthuma de Boer, A. Draaijer, 
and P. M. Houpt, Polymer, 34, 1325 (1993).

18. L. Li, S. Sosnowski, C. E. Chaffey, S. T. Balke, and M. A. Winnik, Langumuir, 10, 2495 (1994).

19. H. Jinnai, Y. Nishikawa, T. Koga, and T. Hashimoto, Macromolecules, 28, 4782 (1995).

20. A. E. Ribbe, T. Hashimoto, and H. Jinnai, J. Matter Sci., 31, 5837 (1996).

21. H. Jinnai, T. Koga, Y. Nisikawa, T. Hashimoto, and S. T. Hyde, Phys. Rev. Lett., 78, 2248 (1997).

22. E. Kumacheva, L. Li, M. A. Winnik, D. M. Shinozaki, and P. C. Cheng, Langmuir, 13, 2483 (1997).

23. O. Vorobyova and M. A. Winnik, J. Polym. Sci., B., 39, 2317 (2001).

24. Y. Terada, S. Hirano, T. Ikehara, and T. Nishi, Polym. J., 33, 371 (2001).

25. N. Loren, A. Altskaer, and A.-M. Hermansson, Macromolecules, 34, 8117 (2001).

26. I. Hopkinson and M. Myatt, Macromolecules, 35, 5153 (2002).

27. T. Magoshi, H. Ziani-Cherif, S. Ohya, Y. Nakayama, and T. Matsuda, Langmuir, 18, 4862 (2002).

28. N. Mashita, T. Minamikawa, K. Uesugi, and H. Jinnai, Kobunshi Ronbunshu, 60, 373 (2003).

29. A. Momose, A. Fujii, H. Jinnai, and H. Kadowaki, Macromolecules, 38, 7197 (2005).

30. S. Koizumi, Y. Yamane, S. Kuroki, I. Ando, Y. Nishikawa, and H. Jinnai, J. Appl. Polym. Sci., 103, 470 (2007).

31. J. Frank, "Electron tomography," Plenum, New York, 1992.

32. R. J. Spontak, M. C. Williams, and D. A. Agard, Polymer, 29, 387 (1988).

33. R. J. Spontak, J. C. Fung, M. B. Braunfeild, J. W. Sedat, D. A. Agard, L. Kane, S. D. Smith, M. Satkowski, A. Ashraf, D. A. Hajduk, and S. M. Gruner, Macromolecules, 29, 4494 (1996).

34. J. H. Laurer, D. A. Hajduk, J. C. Fung, J. W. Sedat, S. D. Smith, S. M. Gruner, D. A. Agard, and R. J. Spontak, Macromolecules, 30, 3938 (1997).

35. J. H. Laurer, A. Ashraf, S. D. Smith, and R. J. Spontak, Langmuir, 13, 2250 (1997).

36. L. H. Radzilowski, B. O. Carragher, and S. I. Stupp, Macromolecules, 30, 2110 (1997).

37. H. Jinnai, Y. Nishikawa, R. J. Spontak, S. D. Smith, D. A. Agard, and T. Hashimoto, Phys. Rev. Lett., 84, 518 (2000).

38. A. J. Koster, U. Ziese, A. J. Verkleij, A. H. Janssen, and K. P. De Jong, J. Phys. Chem. B., 104, 9368 (2000).

39. P. A. Midgley and M. Weyland, Ultramicroscopy, 96, 413 (2003).

40. T. Kaneko, H. Nishioka, T. Nishi, and H. Jinnai, J. Electron. Microsc., 54, 437 (2005).

41. T. Xu, A. V. Zvelindovsky, G. J. A. Sevink, K. S. Lyakhova, H. Jinnai, and T. P. Russell, Macromolecules, 38, 10788 (2005).

42. H. Sugimori, T. Nishi, and H. Jinnai, Macromolecules, 38, 10226 (2005).

43. S. Pratip and N. Jacqes W. M., Macromol. Rapid Commun., 26, 542 (2005).

44. H. Jinnai, K. Sawa, and T. Nishi, Macromolecules, 39, 5815 (2006).

45. H. Nishioka, K. Niihara, T. Kaneko, J. Yamanaka, Y.
Nishikawa, T. Inoue, T. Nishi, and H. Jinnai, Compos. Interfaces, 13, 589 (2006).

46. J. H. Kim, D. H. Lee, J. Won, H. Jinnai, and Y. S. Kang, J. Membr. Sci., 281, 369 (2006).

47. H. Jinnai, H. Hasegawa, Y. Nishikawa, G. J. Agur Sevink, M. B. Braunfeld, D. A. Agard, and R. J. Spontak, Macromol. Rapid Commun., 27, 1424 (2006).

48. H. Jinnai, T. Kaneko, H. Nishioka, H. Hasegawa, and T. Nishi, Chem. Rec., 6, 267 (2006).

49. M. Ono, H. Nishioka, H. Jinnai, K. Nakajima, and T. Nishi, e-J. of Soft Mat., 2, 56 (2006). (URL: http://www.jstage.jst. go.jp/article/ejsm/2/0/2_56/_article/-char/ja/).

50. T. Kaneko, K. Suda, K. Satoh, M. Kamigaito, T. Kato, T. Ono, E. Nakamura, T. Nishi, and H. Jinnai, Macromol. Symp., 242, 80 (2006).

51. H. Jinnai, K. Yasuda, and T. Nishi, Macromol. Symp., 245, 170 (2006).

52. N. Kawase, M. Kato, H. Nishioka, and H. Jinnai, Ultramicroscopy, 107, 8 (2007).

53. H.-W. Park, K. Im, B. Chung, M. Ree, T. Chang, K. Sawa, and H. Jinnai, Macromolecules, 40, 2603 (2007).

54. T. Ikehara, H. Jinnai, T. Kaneko, H. Nishioka, and T. Nishi, J. Polym. Sci., Part B: Polym. Phys., 45, 1122 (2007).

55. Y. Nishikawa, H. Jinnai, T. Koga, T. Hashimoto, and S. T. Hyde, Langmuir, 14, 1242 (1998).

56. Y. Nishikawa, T. Koga, T. Hashimoto, and H. Jinnai, Langmuir, 17, 3254 (2001).

57. Y. Nishikawa, H. Jinnai, and H. Hasegawa, Kobunshi Ronbunshu, 58, 13 (2000).

58. H. Jinnai, H. Watashiba, T. Kajihara, and M. Takahashi, J. Chem. Phys., 119, 7554 (2003).

59. A $360^{\circ}$ single-axis tilt stage was used for $\pm 90^{\circ}$ rotation in the past (D. P. Barnard et al. Microscopy 167, 39 (1992)). However, it is for high-voltage electron microscope and is NOT versatile.

60. S. Hyde, S. Andersson, K. Larsson, Z. Blum, T. Landh, S. Lidin, and B. W. Niham, in "The language of shape," Elsevier, Amsterdam, 1997.

61. a) K. Sawa, H. Jinnai, H. W. Park, T. Chang, and T. Nishi, Polym. Prepr. Jpn., 55, 3588 (2006).

b) H. Jinnai, K. Sawa, F. Hirato, H. W. Park, T. Chang, and T. Nishi, manuscript in preparation.

62. a) H. Sugimori, U. Matsuwaki, H. Morita, M. Doi, T. Nishi, and H. Jinnai, Polym. Prepr. Jpn., 55, 1321 (2006).

b) H. Sugimori, U. Matsuwaki, H. Morita, M. Doi, T. Nishi, and H. Jinnai, manuscript in preparation.

63. Y. Shinbori, T. Kitaoka, H. Sugimori, Y. Nishikawa, H. Jinnai, K. Akutagawa, N. Mashita, and T. Nishi, Polym. Prepr. Jpn., 55, 994 (2006).

64. H. Jinnai, Y. Shinbori, T. Kitaoka, K. Akutagawa, N. Mashita, and T. Nishi, Macromolecules, submitted.

65. T. Kitaoka, Y. Shinbori, H. Sugimori, Y. Nishikawa, H. Jinnai, K. Akutagawa, N. Mashita, and T. Nishi, Polym. Prepr. Jpn., 55, 3340 (2006).

66. A. Lapra, F. Clement, L. Bokobza, and L. Monnerie, Rubber Chem. Technol., 76, 60 (2004).

67. Due to the missing wedge problem in TEMT experiments, areas that appear to be pale perpendicular to the tilt axis can be artifacts in the 3D reconstruction. The tilt axis is par- 
allel to Y-axis in Figure 5. Thus, the half-bright zones discussed in the text should truly correspond to actual thickness of the rubber matrix.

68. W. Reichert, D. Göritz, and E. J. Duschl, Polymer, 34, 1216 (1993).

69. H. Dohi, Polym. Prepr. Jpn., 53, 4255 (2004).
70. H. Dohi, M. Sakai, Y. Minagawa, and H. Kishimoto at the 52th Rheology Conference, Hirosaki Japan, September 2224, 2004.

71. J. Y. Lee, Q. Zhang, T. Emrick, and A. J. Crosby, Macromolecules, 39, 7392 (2006).

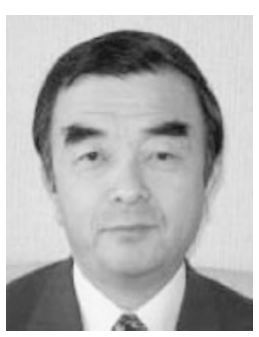

Hidehiko DOHI graduated from the Department of Petro-Chemistry of Kyoto University, Kyoto, Japan in 1973. In the same year, he got employment in Sumitomo Rubber Industries, Ltd., Kobe, Japan. In 1998, he moved to General Manager of Chemical Analysis Center in Research and Development Division of Sumitomo Rubber Industries, Ltd. In 2003, he moved to General Manager of Chemical Analysis Center of SRI R\&D Ltd., because of the organizational reform of the company. His research interest is the development of new analysis technique of rubber materials. He is also a graduate student at the Department of Biotechnology, Tokyo University of Agriculture and Technology. His research theme is the study on nano-structures in rubber.

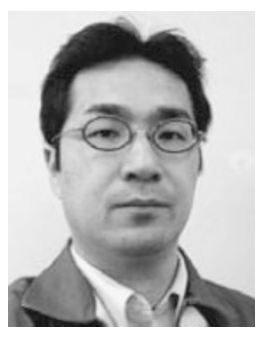

Hideaki KIMURA was born in Miyagi, Japan, in 1969. He awarded Doctor of Engineering in 1998 at Gunma University. He was a principal scientist at JCII (Japan Chemical Innovation Institute) as a NEDO (New Energy and Industrial Technology Development Organization) fellowship researcher from 1998 to 2001. He received a JSPS (Japan Society for the Promotion of Science) Research Fellowships for Young Scientists from 2001 to 2004. During this period, he studied at Tokyo Institute of Technology and Iowa State University, USA. He was a guest scientist at Tsukuba University in 2005. His present position is the associate manager of the chemical analysis center of SRI R\&D Ltd. His research interests are the molecular structure and dynamics of rubbers and resins.

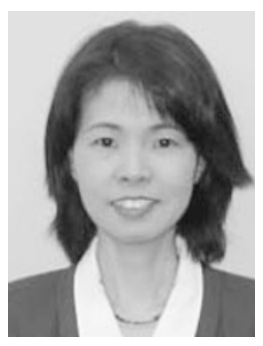

Marina KOTANI got employment in Sumitomo Rubber Industries, Ltd., Kobe, Japan. She worked for Tire Material Research Division. She moved to Manager of Chemical Analysis Center of SRI R\&D Ltd., because of the organizational reform of the company. Her research interest is rubber analysis.

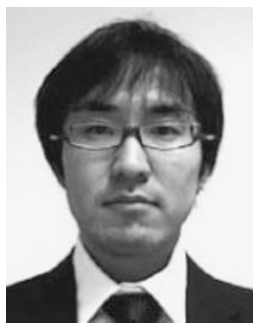

Takeshi KANEKO was born in Chiba, Japan in 1979 and grew up in Osaka, Japan. He obtained his education in polymer science from the Department of Mcromolecular Science and Engineering, Kyoto Institute of Technology, where he received his B.S. degree in 2003 and his M.S. degree in 2005. He is interested in three-dimensional observation and analysis of microphase-separated structures of block copolymers.

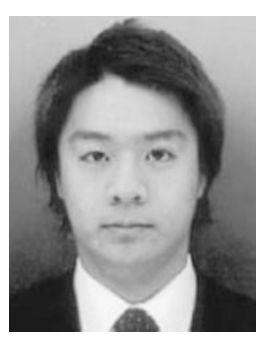

Tatsuro KITAOKA was born in Hyogo, Japan in 1984. He obtained his education in polymer science from the Department of Macromolecular Science and Engineering, Kyoto Institute of Technology, Kyoto, Japan where he received his B.S. degree in 2006. He is interested in 3D observation and structural analysis of nanocomposite materials. 


\section{H. DOHI et al.}
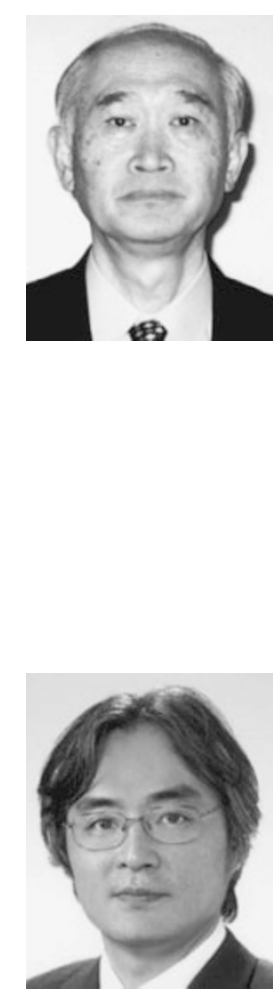

Hiroshi JINNAI was born in Osaka, Japan, in 1965, and graduated from the Department of Polymer Chemistry of Kyoto University, Kyoto, Japan in 1988. He studied polymer physics and awarded Master of Engineering in 1990 and Doctor of Engineering in 1993 at the same university. During this period, he was a guest scientist at National Institute of Standards and Technology (NIST), USA from 1988 to 1989 and from 1991 to 1992. He received a JSPS (Japan Society for the Promotion of Science) Research Fellowships for Young Scientists in 1993. In 1993, he joined an ERATO (Exploratory Research for Advanced Technology) program, Hashimoto Polymer Phasing Project, as a group leader. He was appointed lecturer at the Department of Macromolecular Science and Engineering of Kyoto Institute of Technology in 1998 and was promoted to an associate professor in 2002. He was awarded the SPSJ (The Society of Polymer Science, Japan) Wiley Award in 2006. His research interests are the self-assembling morphologies and processes of polymer blends and block copolymers. He is also a recognized world expert in three-dimensional visualization of soft-condensed matters. 